BULL. AUSTRAL. MATH. SOC.

VOL. $2(1970), \mid 25-128$.

\title{
Orthogonality relations and orthomodularity
}

\author{
P. D. Finch
}

An abstract orthogonality relation is defined, a closure operation and a corresponding lattice of closed sets are associated with it. Necessary and sufficient conditions are obtained for the orthomodularity of a sub-ortholattice of the lattice of closed sets.

\section{Introduction}

A binary relation $\perp$ on a non-empty set $I$ is said to be an orthogonality relation when

(1) $x \perp y$ implies $y \perp x$,

(2) $x \perp x$ implies $x \perp y$ for all $y$ in $I$.

Note that a particular case of (2) occurs when 1 is anti-reflexive, that is $x \perp x$ for no $x$ in $I$.

REMARK. In [3] MacLaren defines an orthogonality relation by requiring that

(3) ( $z \perp x$ if and only if $z \perp y$ ) implies $x=y$, in addition to (1) and (2) above. However, we make no use of (3) and so we omit it from the definition of an orthogonality relation.

An orthogonality relation is a special kind of polarity in the sense of Birkhoff [1], p. 122-3, and it follows from the results given there that $X \rightarrow X^{\perp}$ is a closure operation on the subsets of $I$, and that $X \rightarrow X^{\perp}$ is an orthocomplementation of the lattice of closed subsets of 
$I$. Here, of course,

$$
X^{\perp}=\{y: y \perp x, \forall x \in X\}
$$

We write $I^{\perp}=0$. Note that 0 is the empty set when $\perp$ is anti-reflexive; when 0 is not the empty set one has

$$
0=\{x: x \in L \& x \perp y, \forall y \in L\} \text {. }
$$

A subset $S$ of $I$ is said to be an orthogonal set when $x \perp y$ for any $x, y$ in $S$ with $x \neq y$. In particular the set 0 and the one element subsets of $I$ are orthogonal sets. Let $A$ be a subset of $I$ and let $P$ be an orthogonal subset of $A$; a straight-forward argument establishes that the set of all orthogonal subsets of $A$ which contain $P$ has at least one element which is maximal with respect to set inclusion.

Let $L$ denote the lattice of closed subsets of $I$. By a sub-ortholattice of $L$ we mean a sublattice $L$ of $L$ which contains $I$ and has the property that $X^{\perp}$ is in $L$ whenever $X$ is in $L$. A sub-ortholattice $L$ of $L$ is clearly orthocomplemented, our interest here is the determination of necessary and sufficient conditions for its orthomodularity, that it has the property (cf. Birkhoff [1])

$$
X \subseteq Y^{\perp} \& X \vee Y=I \Rightarrow X=Y^{\perp}
$$

for any $X, Y$ in $L$. Note that orthocomplementation of (1) gives

$$
X \geq Y^{\perp} \& X \cap Y=0 \Rightarrow X=Y^{\perp} \text {, }
$$

we use this fact below.

\section{Orthomodularity in a sub-ortholattice}

We say that a sub-ortholattice $L$ of $L$ has the $B$-property when, for each $X$ in $L$ and any maximal orthogonal subset $M$ of $X$ one has $M^{\mathcal{L}}=X$. We note firstly,

LEMMA. If $X$ is a closed subset and $M$ is a maximal orthogonal subset of $X$ then $0 \subseteq M$.

Proof. Since $X$ is closed it contains 0 , if $P \subseteq X$ is orthogonal then $P \cup O \subseteq X$ is also orthogonal. 
We are now able to prove our main result, namely

THEOREM. A sub-ortholattice $L$ of $L$ is orthomodular if and only if it has the B-property.

Proof. Suppose that $L$ does have the $B$-property and assume the antecedent in the implication (1). We prove that $X=y^{\perp}$. To do so let $M$ be a maximal orthogonal subset of $X$ and let $P$ be a maximal orthogonal subset of $Y$. Then

$$
I=X \vee Y=\left(M^{L \perp} \cup P^{\perp \perp}\right)=(M \cup P)^{\perp \perp} \text {. }
$$

It follows that $M \cup P$ is a maximal orthogonal subset of $I$ for

$$
x \perp M \cup P \Rightarrow x \in(M \cup P)^{\perp}=0
$$

and $0 \subseteq M \cup P$ by the lemma.

Let $N \supseteq M$ be a maximal orthogonal subset of $Y^{\perp}$ which contains $M$, then $N \bar{\perp} P$ and

$$
(N \cup P)^{\perp \perp}=\left(Y^{\perp} \cup Y\right)^{\perp \perp}=I \text {. }
$$

Since $M \cup P$ is maximal and $M \cup P \subseteq N \cup P$ we must have $M=N$, that is $X=M^{\perp L}=N^{\perp \perp}=Y$. This establishes that $L$ is orthomodular. Conversely assume that $L$ is orthomodular, we establish that it has the $B$-property. Let $X$ be in $L$ and let $M$ be a maximal orthogonal subset of $X$. Since $M$ is maximal we have $M^{\perp} \cap X=0$, but $X \geq M^{\perp \perp}$ and so, using orthomodularity in the form (2), we have $X=M^{L L}$, that is $L$ has the $B$-property.

REMARK. Note that the theorem remains meaningful when $L=L$. In fact a study of the proof shows that we do not need to assume that $L$ is a lattice, the theorem remains valid when $L$ is a sub-orthoposet of $L$ in which orthogonal joins exist. A special case of the theorem, namely when $L=L$ and $L$ is the completion by cuts of an orthoposet was established in Finch [2]. 


\section{References}

[1] Garrett Birkhoff, Lattice theory (Colloquium Publ. 25, Amer. Math. Soc., Providence, 3rd ed., 1967).

[2] P.D. Finch, "On orthomodular posets", J. Austral. Math. Soc. (to appear).

[3] M. Donald MacLaren, "Atomic orthocomplemented lattices", Pacific J. Math. 14 (1964), 597-612.

Monash University,

Clayton, Victorla. 